\title{
Medicina de Precisión: Aspectos Prácticos para el Cirujano Moderno
}

\author{
Carlos A. Vaccaro, Marcos González, Nadia C. Peralta \\ Hospital Italiano de Buenos Aires. C.A.B.A., Argentina
}

Los contenidos de este capitulo se basan en la $3^{a}$ edición de las Clínicas Quirúrgicas de Cáncer Colorrectal. C. Vaccaro y N. Peralta. del hospital ediciones 2020 (en prensa).

\section{INTRODUCCIÓN}

Actualmente se considera que el CCR no es una sola entidad, sino un grupo de neoplasias con gran heterogeneidad tanto a nivel intertumoral como intratumoral. Dada la compleja composición molecular de estos tumores, en los últimos años los esfuerzos se han centrado en la identificación de posibles marcadores moleculares que proporcionen información sobre la respuesta del tumor a terapias anticancerígenas, lo que a su vez ayudaría a personalizar el tratamiento para el CCR. Los biomarcadores "predictivos" identifican pacientes que tienen más probabilidades de beneficiarse de un tratamiento, y por lo tanto pueden desempeñar un papel crítico en guiar las decisiones de tratamiento.

En este artículo se describirán los avances más recientes y aplicados en la estratificación molecular del CCR con utilidad clínica.

\section{¿QUÉ ES LA MEDICINA DE PRECISIÓN?}

La medicina de precisión tiene como objetivo personalizar la atención médica con decisiones y tratamientos adaptados a cada individuo. Aunque el análisis genómico es todavía un desarrollo relativamente nuevo en el tratamiento de drogas, este campo se está expandiendo rápidamente. En la actualidad, más de 200 medicamentos tienen información en sus etiquetas sobre biomarcadores farmacogenómicos, información genética mensurable o identificable que se puede utilizar para individualizar el uso de un medicamento.

En el CCR, la Medicina de Precisión se centra en los biomarcadores predictivos. Estos son factores biológicos que brindan información sobre la probabilidad de respuesta tumoral a un determinado agente terapéutico (a diferencia del factor pronóstico que proporciona información prospectiva de la evolución del paciente). En el CCR

Carlos Vaccaro

carlos.vaccaro@hospitalitaliano.org.ar

Recibido: Marzo de 2020. Aceptado: Mayo de 2020 estos biomarcadores están relacionados a los genes involucrados en la carcinogénesis colorrectal y sus mutaciones.

La utilización de estos biomarcadores ya son estándares de tratamiento en el CCR metastásico. Es necesario que todo médico involucrado en el manejo de estos pacientes tenga el conocimiento básico que le permita participar activamente en la toma de decisión en los equipos multidisciplinarios.

\section{IMPACTODELAHETEROGENEIDADTUMORAL}

El CCR es consecuencia de una acumulación de mutaciones que le confieren una ventaja selectiva de crecimiento local en relación con las células normales y la capacidad de dar metástasis. Si bien existen cientos de mutaciones, algunas de ellas son determinantes para los procesos de inicio, progresión y metástasis y se las conoce como "drivers" (conductoras) para diferenciarlas de las mutaciones secundarias o "passengers" (pasajeras) mucho más numerosas, pero no influyentes en los procesos mencionados. Cada tumor tiene entre 2 a 6 mutaciones conductoras en los genes APC (en 80\% de los tumores), TP53 (50\%), KRAS (35\%-45\%), PIK3CA (20\%-30\%), y BRAF (10\%).

El CCR es una enfermedad que presenta una gran heterogeneidad tumoral en varios niveles diferentes. En primer lugar, la composición genética de un tipo de tumor dado puede variar significativamente entre un paciente $\mathrm{y}$ otro. También dentro de un paciente individual, existe un alto grado de heterogeneidad genética entre el tumor primario y la metástasis, así como entre los diferentes sitios metastásicos (heterogeneidad intertumoral). La heterogeneidad puede existir en diferentes áreas dentro de un mismo tumor (heterogeneidad intratumoral), o en diferentes regiones del mismo paciente (heterogeneidad tumoral espacial). También puede darse en diferentes momentos del mismo tumor (heterogeneidad tumoral temporal) secundaria a la capacidad de diferentes clones para resistir las terapias.

Estos diferentes niveles de heterogeneidad tumoral pueden afectar la señalización de múltiples vías oncogénicas claves en una variación fenotípica extensa, de tal manera que cada tumor o incluso dentro del mismo tumor muestra su propio patrón genético, epigenético, transcriptómico y proteómico. En consecuencia, la heterogeneidad intratumoral plantea un enorme desafío a la práctica de la medicina personalizada porque un agente terapéutico de- 
terminado dirigido a un agente molecular específico es probable que sólo impacte en una minoría de pacientes.

\section{CARCINOGÉNESIS COLORRECTAL: INESTABILIDAD MICROSATELITAL Y SUS IMPLICANCIAS}

Existen dos tipos de inestabilidad genómica: el tipo más común es la inestabilidad cromosomal (presente en el 80$85 \%$ de los CCR esporádicos) que es un mecanismo muy eficiente en causar alteraciones en genes como APC, P53 y SMAD4. El otro tipo de inestabilidad es la microsatelital (IMS) que, si bien es característica de los tumores del síndrome de Lynch, está presente en alrededor del 15\% de los tumores esporádicos y tiene importantes implicancias pronósticas y predictivas de respuesta al tratamiento.

La IMS se considera huella molecular de un defecto en la reparación de los errores que se producen durante la duplicación del ADN (DNA mismatch-repair defects). E1 sistema que repara estos defectos está integrado por los genes de reparación MLH1, MSH2, MSH6 y PMS2. Estos genes pueden afectarse por mutaciones germinales heredables (produciendo el síndrome de Lynch) y en el caso exclusivo del MLH1 por una metilación no heredable que produce su inactivación. En ambos casos se produce una acumulación de errores de apareo de bases en regiones denominadas microsatélites, afectando fundamentalmente a genes como el TGF-BR2 (transforming growth factor b receptor type II) y BAX (BCL2-associated $\mathrm{X}$ protein).

Los CCR con IMS se localizan más frecuentemente en el colon proximal, son poco diferenciados y tienen histología mucinosa o medular. Su diagnóstico se basa en el estudio inmunohistoquímico del tejido tumoral de las proteínas codificadas por los genes de reparación MLH1, MSH2, MSH6 y PMS2.

Estos tumores también son considerados altamente inmunogénicos dada la alta carga mutacional asociada a ellos que redunda en la formación de neoantígenos específicos del tumor e infiltración de células T. Esto explica la observación de que la IMS se asocia con pronóstico favorable en pacientes con cáncer de colon en estadio temprano y que se asocie a una mayor sensibilidad a los nuevos agentes inmunoterápicos como el anti PD-1 pembrolizumab y nivolumab.

En un estudio de fase II con pembrolizumab la tasa de control de enfermedad fue de $89 \%$ en el grupo con IMS y de $16 \%$ en los tumores sin IMS. El estudio CheckMate con 45 pacientes comprobó la eficacia en primera línea del nivolumab con baja dosis de Ipilimumab en CCR metastásico con MSI-H/dMMR. La tasa de respuesta objetiva fue del 60\%, la tasa de control de la enfermedad fue del $84 \%$ y el $7 \%$ de los pacientes tuvieron una respuesta completa. A 12 meses las tasas de sobrevida libre de progresión y sobrevida global fueron del 77\% y 83\%, respectivamente. Basado en esta evidencia y a pesar del pequeño tamaño de estos estudios, la FDA ha aprobado al pembrolizumab y nivolumab para su uso en tumores inestables que han progresado luego de quimioterapia.

\section{MUTACIÓN V600E DEL GEN BRAF}

El estudio del gen BRAF es una de las determinaciones más frecuentemente utilizada cuando se detecta inestabilidad microsatelital (más concretamente déficit de expresión del gen de reparación MLH1). La mutación V600E representa más del $80 \%$ de las alteraciones, lo que facilita el estudio y disminuye los costos. Cuando el BRAF está mutado se produce una inactivación (adquirida a través de una metilación aberrante) del MLH1 y el tumor adquiere inestabilidad microsatelital. La presencia de una mutación V600E es mutuamente excluyente de una mutación germinal del MLH1 por lo que su detección prácticamente descarta al síndrome de Lynch. Además de esta importante implicancia diagnóstica, la mutación BRAF V600E es un factor predictivo a la respuesta al panitumumab y cetuximab la cual es altamente improbable salvo que se administren inhibidores del BRAF (esquemas todavía no aprobados para el CCR).

\section{K-RAS}

La familia de genes RAS se compone de tres genes: KRAS, NRAS y HRAS. Las mutaciones de KRAS están presentes en el 30\% -45\% de los CCR (exones 2, 3 y 4). Otro $5 \%$ de los CCR presentan mutaciones activadoras en NRAS (en los mismos exones que KRAS). El papel de las mutaciones de RAS como marcador predictivo negativo para la terapia anti-EGFR en el tratamiento de CCRm ha sido comprobado en múltiples ensayos clínicos. Los estudios han también estableció claramente que la adición de la terapia anti-EGFR (ej. Cetuximab y $\mathrm{Pa}$ nitumumab) a la quimioterapia no mejora los resultados e incluso es potencialmente dañino.

\section{PERSPECTIVAS FUTURAS}

\section{Nuevos biomarcadores emergentes}

Existe una gran variedad de biomarcadores que se están evaluando actualmente y todavía no cuentan con evidencia para la aplicación clínica. La mutación del PIK3CA (presente en el 10-18\% de los CCR) cuenta con evidencia para ser considerada un predictor negativo de respuestas a los agentes anti EGFR. Por su parte la amplificación del 
HER2 tiene un potencial valor predictivo pero su prevalencia es extremadamente baja y el valor de la pérdida de expresión PTEN y de CDX2 es controvertida. Si bien es de esperarse que los subtipos moleculares pueden ser predictivos no se cuenta todavía con ninguna valoración. Los micro RNA (miRNAs) son secuencias no codificantes cortas (18-22 nucleótidos) involucradas en la regulación de la expresión génica y probablemente en la tumorigenesis colorrectal y también están siendo evaluados como factores predictivos o pronósticos.

\section{Biopsia líquida}

Las nuevas tecnologías permiten aislar diferentes componentes tumorales circulantes en la sangre (ej. células tumorales, ADN tumoral, exosomas, microARN) que pueden proporcionar información útil para fines diagnósticos, de pronóstico y predictivos. El estudio de la biopsia líquida ha adquirido un lugar prominente en la investigación del cáncer (especialmente en pulmón), y una de las características que lo hace especialmente prometedor es su capacidad para superar el problema de la heterogeneidad tumoral. A diferencia del tejido tumoral que, por ser heterogéneo, su biopsia puede no ser representativa de todo el tumor, la biopsia líquida no solo puede ser más representativa, sino que permite seguir la evolución del tumor en tiempo real. En un estudio realizado por Morelli et al. el análisis del plasma recogido en 60 pacientes refractarios a anti-EGFR mAbs y sin mutación inicial del KRAS reveló la presencia de mutación KRAS en el 44\% de los casos. Estas nuevas mutaciones detectadas en pacientes refractarios al tratamiento anti-EGFR puede derivar de clones raros y preexistentes en tumores primarios.

\section{E1 Enfoque Multiómico}

Las técnicas "omicas" a gran escala tienen la capacidad de

\section{BIBLIOGRAFÍA}

1. Guler I, Askan G, Klostergaard J, Sahin IH. Precision medicine for metastatic colorectal cancer: an evolving era. Expert Rev Gastroenterol Hepatol. 2019;13: 919-931.

2. Sandhu J, Lavingia V, Fakih M. Systemic treatment for metastatic colorectal cancer in the era of precision medicine. Journal of Surgical Oncology. 2019. pp. 564-582.

3. Korphaisarn K, Kopetz S. BRAF-Directed Therapy in Metastatic Colorectal Cancer. Cancer J. 2016;22: 175-178.

4. Henry JT, Johnson B. Current and evolving biomarkers for precision oncology in the management of metastatic colorectal cancer. Chinese Clinical Oncology. 2019. pp. 49-49.

5. Morris VK. Systemic Therapy in BRAF V600E-Mutant Metastatic Colorectal Cancer: Recent Advances and Future Strategies. Current Colorectal Cancer Reports. 2019. pp. 53-60.

6. Wilson A, Ronnekliev-Kelly S, Winner M, Pawlik TM. LiverDirected Therapy in Metastatic Colorectal Cancer. Current Colorectal Cancer Reports. 2016. pp. 67-80.

7. Maurel J, Maurel JM. EGFR Blockade as Effective Therapy in identificar la biología tumoral con una definición extraordinariamente alta que permite la comprensión de patologías complejas y discriminar tumores con fenotipos moleculares distintos. La transcriptómica es el estudio de la expresión global del ARNm de un tejido en particular. La epigenómica es el estudio de modificaciones epigenéticas que tienen un rol importante en la carcinogénesis y son frecuentes en el CCR. Estas alteraciones incluyen la metilación de la región promotora del ADN y las modificaciones en las histonas. La proteómica estudia proteínas a gran escala para mapear procesos biológicos involucrados en la carcinogénesis. Por último, la genómica, basada en los avances en la tecnología de secuenciación de próxima generación (Next Generation Sequencing - NGS) permite el estudio de millones de bases de ADN. Esta metodología proporciona una gran oportunidad para en desarrollo de biomardores aplicables a la medicina de precisión.

\section{Epidemiología Molecular}

Además de la heterogeneidad que caracteriza al cáncer colorrectal debido a su notable inestabilidad genética, otra gran barrera para el desarrollo de la medicina de precisión es la acción de los factores exógenos como el estilo de vida, la dieta, la nutrición, el medio ambiente y el microbioma. Estos factores también influyen en la patogénesis y comportamiento del CCR accionando sobre, no solo las células tumorales, sino también en las células no neoplásicas, incluidas las células inmunitarias (micromedioambiente). De allí la importancia de investigar los efectos interactivos entre los tumores y la totalidad de las exposiciones (llamadas exposomas). Esto se ha convertido en un nuevo campo de investigación conocido como epidemiología molecular cuyo objetivo es proporcionar nuevos conocimientos sobre las interacciones entre el ambiente, el tumor y el huésped para entender mejor cómo los factores endógenos y exógenos modifican los fenotipos tumorales.

BRAF and EGFR Mutated Metastatic Colorectal Cancer: Learning from a Clinical Case. Austin Journal Of Surgery. 2019.

8. Ålgars A, Sundström J, Lintunen M, et al. EGFR gene copy number predicts response to anti-EGFR treatment in RAS wild type and RAS/BRAF/PIK3CA wild type metastatic colorectal cancer. Int J Cancer. 2017;140: 922-929.

9. Puzzoni M, Giampieri R, Demurtas L, et al. The role of sidedness, EGFR gene copy number (GCN) and EGFR promoter methylation in RAS/BRAF wild type (WT) colorectal cancer (CRC) patients receiving irinotecan/cetuximab. Journal of Clinical Oncology. 2017. pp. 628-628.

10. Lièvre A, Ouine B, Canet J, et al. Correction: Protein biomarkers predictive for response to anti-EGFR treatment in RAS wild-type metastatic colorectal carcinoma. British Journal of Cancer. 2018. pp. 387-387.

11. Tie J, Desai J. Targeting BRAF mutant metastatic colorectal cancer: clinical implications and emerging therapeutic strategies. Target Oncol. 2015;10: 179-188. 